\title{
Análisis factorial múltiple para clasificación de universidades latinoamericanas
}

\author{
Multiple Factor Analysis for Ranking Latinamerican Universities
}

\author{
Jimmy Antonio Corzo Salamance
}

jacorzos@unal.edu.co

\begin{abstract}
Resumen
Se utiliza la técnica del análisis factorial múltiple (AFM) para proponer cinco clases de universidades latinoamericanas a partir de tres conocidos rankings, los cuales distinguen, por ejemplo: universidades con alto grado de especialización y baja productividad, universidades de excelencia con bajo liderazgo científico, universidades que no gozan de buena reputación, pero tienen docentes productivos, universidades con buena reputación y pocos docentes con doctorado y universidades productivas de alto impacto y bajos índices de colaboración internacional. Los resultados del AFM revelan algunas paradojas que se corroboran en la clasificación como el hecho de oponer el grado de especialización a la productividad, el liderazgo científico contra impacto y calidad de la productividad, o la posible inconveniencia de incluir criterios de opinión que resultan independientes del liderazgo y del impactd ${ }^{1}$
\end{abstract}

Palabras clave: Análisis factorial múltiple, rankings de universidades, clasificación de universidades.

\begin{abstract}
We use the Multiple Factor Analysis (MFA) to built five classes of Latinamerican Universities from three known university rankings. These classes distinguish among universities with high level of specialization and low academic output, universities of excelence with low Scientific Leadership, universities with no good reputation and productive staff, universities with good reputation and few doctoral staff, and productive universities with high impact and low indicators of international collaboration. The factors produced by the MFA reveal some paradoxes corroborated in the classification by the fact that they counterpose the level of specialization vs. Productivity, the scientific leadership vs. impact and quality of the output, and
\end{abstract}

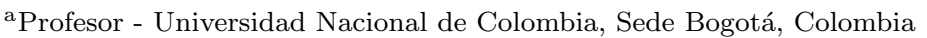

${ }^{1}$ Agradezco al Estadístico Jairo Jimenez por su apoyo en el arreglo de la base de datos.
} 
they reveal too the possible inconvenience to include judging criteria, which result independent of leadership and impact.

Keywords: Multiple Factor Analysis, University rankings, Universities Classification.

\section{Introducción}

Los rankings son clasificaciones de universidades basadas en indicadores de criterios académicos e investigativos y, en algunos casos, también en percepciones sobre el prestigio de las universidades. El primero que se puede catalogar en esta definición fue publicado en 1998 por el Centrum für Hochschulentwicklung (CHE) con el mismo nombre. Su objetivo era proporcionar información a aspirantes y estudiantes acerca de maestrías y doctorados, para mostrar indicadores de internacionalización de un grupo seleccionado de universidades europeas. Desde entonces ha sido notoria su proliferación, al punto de que, por ejemplo, Sanchez \& Moreno (2011) reportaron 21 rankings, la mayoría de ellos surgidos desde 2007 a la fecha. Una interesante nota histórica sobre los comienzos de los rankings alrededor de 1900 se encuentra en Martinez Rizo (2011).

Los criterios utilizados en los rankings, así como las ponderaciones asignadas a ellos, varían naturalmente de acuerdo con los objetivos del ranking, con los campos temáticos a los que se refieren, con las regiones geográficas para las cuales son construidos y por supuesto con los intereses de las entidades que los construyen. Por otra parte, todos contienen un modelo implícito de universidad definido por la entidad productora del ranking, el cual no necesariamente coresponde a intereses o necesidades académicas de las instituciones. No obstante, la mayoría de ellos incluyen al menos tres criterios comunes realcionados con la productividad (institucional o de los docentes-investigadores), su impacto y su calidad.

Muchos han sido los cuestionamientos a dichos rankings, a los criterios o indicadores utilizados, al modelo de universidad implícito, a su pertinencia cuando se utilizan en decisiones sobre asignación de recursos, al hecho de estar basados en publicaciones en revistas de habla inglesa, a la exclusión o baja importancia asignada a la investigación en ciencias sociales, a la dependencia (de algunos de los indicadores) del tamaño de las instituciones etc. véase Margison \& Ordorika (2010) o Martinez Rizo (2011). Adicionalmente, el interés institucional por obtener los mejores puestos en los rankings ha generando el riesgo de que las universidades se vean forzadas a reorientar sus paradigmas de investigación con tal de posicionarse bien en dichos rankings, abandonando temas de interés local, regional o nacional. 
Para contribuir a esta discusión se analizan similitudes y diferencias entre tres rankings (Scimago, Webometrics y QS), los cuales producen periódicamente información sobre universidades latinoamericanas. Para ilustrar el uso del AFM se propone en la primera sección una clasificación basada en los indicadores de los tres rankings simultáneamente.

En la segunda sección se hace un breve presentación de los criterios utilizados en los tres rankings untilizados en este análisis, en la tercera sección, una rápida presentación de la metodología, en la cuarta sección, los principales resultados, y en la quinta sección, las conclusiones y una breve discusión sobre los resultados.

\section{Descripción de tres clasificaciones que utilizan rankings}

Para este ejercicio se escogieron tres de los rankings que acopian y publican información sobre universidades latinoamericanas periódicamente y que comparten parcialmente algunos de los criterios utilizados para su construcción.

\section{Ranking web de universidades (Web)}

También llamado como Webometrics, se le reconoce como un indicador de presencia institucional en la web. Está construido con cuatro indicadores: Impacto en la web (ponderado con el $50 \%$ para el cálculo del ranking ) medido por el número de enlaces entrantes al dominio de la universidad; presencia (16.66\%) que es el número de páginas web alojadas en el dominio de la universidad; apertura $(16.66 \%)$ que corresponde al número de archivos enriquecidos en formatos pdf, doc, docx, ppt, publicados en sitios web de la institución; excelencia (16.66\%) medida por los trabajos académicos publicados en revistas internacionales que están en el $10 \%$ de los artículos más citados (obtenido del ranking Scimago).

\section{Scimago Institutions Ranking (Sci)}

Se autodefine como un indicador de evaluación de la actividad investigadora de universidades e instituciones de investigación. Utiliza tres grupos de indicadores: 


\section{Indicadores de investigación:}

- Productividad institucional: productividad ${ }^{2}$ medida por el número de artículos de la institución, publicados en revistas indexadas en Scopus;

- Colaboración internacional: producción de la institución en colaboración con instituciones extranjeras;

- Impacto normalizado: indicador de sobre o subcitaciones de la producción de una institución con respecto al número de citaciones de la producción mundial:

$$
I N=\frac{\text { Impacto promedio de la producción científica de una institución }}{\text { Impacto promedio mundial }}
$$

Donde el impacto se mide por el número de citaciones de la producción científica. Por ejemplo $I N=1.2$ significa que la producción científica de la institución es citada un $20 \%$ por encima del promedio mundial de citaciones, mientras que $I N=0.7$ indica que la productividad de la institución es citada $30 \%$ por debajo del promedio de citaciones de la productividad mundia 3 . Aquí es importante diferenciar:

- Publicaciones de alta calidad: proporción de publicaciones de una institución en revistas rankeadas en el primer cuartil del Scimago Journal Rank.

- Índice de especialización: indica grado de concentración o dispersión temática de la producción científica de una institución y se calcula como el índice de Gini.

- Excelencia: calculada como el porcentaje de producción científica de una institución ubicada en el $10 \%$ de los papers más citados en su área.

- Liderazgo científico: porcentaje de producción de una institución en la cual esta es el principal contribuidor.

- Excelencia con liderazgo: que indica el número de documentos de excelencia en los cuales el principal contribuyente es la institución.

- Talento científico: corresponde al número de autores de la institución en la producción total de ésta.

\section{Indicadores de innovación}

- Conocimiento innovador: medido por la producción científica de una institución citada en patentes.

\footnotetext{
${ }^{2}$ Que se distingue de la productividad docente utillizada en el Qs donde se mide por el número de artículos por docente.

${ }^{3}$ Tomado del ítem «oriented field normalized citation score average» producido por el Karolinska Intitutet de Suecia
} 
- Impacto tecnológico: indica el porcentaje de publicaciones científicas citadas en patentes.

\section{Indicadores de presencia en la web}

- Tamaño de la web: que corresponde al número de páginas asociadas a la URL de una institución según el buscador Google (https://www.google.com)

- Enlaces entrantes al dominio de la institución: que es el número de enlaces entrantes al dominio de la institución tomado de la conocida herramienta de análisis de enlaces Ahrefs (https://ahrefs.com).

\section{Ranking de universidades QS}

Se presenta con la pretensión de mostrar las mejores universidades de América Latina basado en siete indicadores: 1 . Reputación entre pares académicos obtenido por medio de una encuesta, 2. Reputación entre empleadores, que también se obtiene por medio de una encuesta, 3 . Número de estudiantes por profesor, 4 . Número de profesores con doctorado, 5. Número promedio de citas por artículo publicado, tomado de Scopus, 6. Promedio de artículos publicados por docente, tomado de Scopus, 7. Impacto en la web, tomado del Webometrics.

Las ponderaciones de los criterios utilizados por este ranking han variado con los años. Por ejemplo, para el año 2014, los dos indicadores de reputación tenían ponderaciones de $30 \%$ y $20 \%$ respectivamente, y los otros cinco tenían ponderaciones de $10 \%$ cada uno, mientras que para el año 2012 las ponderaciones eran 40 y 10 para las reputaciones entre pares académicos y empleadores respectivamente y $20 \%, 20 \%, 5 \%, 5 \%$ para los otros cuatro criterios respectivamente. Lo anterior induce cierta inestabilidad y hace incomparables los valores del indicador por años.

\section{Metodología}

El AFM es una técnica de análisis de datos multivariados que generaliza el análisis de componentes principales (ACP), se aplica en contextos en los que se han observado grupos de variables de naturaleza diferente sobre un mismo conjunto de objetos, de manera que no resulta adecuado llevar a cabo un ACP de todos los grupos mezclados. Otro caso en el que se utiliza el AFM es cuando los grupos tienen grandes diferencias en el número de variables, en cuyo caso dominarían en el $\mathrm{ACP}$ aquellos grupos de variables en los que haya número más elevado de ellas. La técnica de AFM consiste básicamente en realizar ACP separados por los grupos de variables, utilizar unos indicadores de características compartidas por los grupos 
de variables (coeficientes $L_{g}$ ) para seleccionar de los análisis separados los factores compartidos. Al final se realiza un ACP global sobre los factores compartidos con el cual se identifican asociaciones entre los temas incluidos en los grupos de variables. Uno de los compendios completo de la teoría del AFM es el de Escofier (2008)

La base de datos utilizada es la fusión (intersección) de tres bases de datos obtenidas de las páginas web de los tres rankings y contiene las 150 universidades que ocuparon alguna de esas posiciones según el ranking Qs. Debido a que los criterios de presencia en la web de Scimago y Qs son tomados directa o indirectamente del Webometrics o de sus mismas fuentes, dichos criterios se excluyeron de estos dos rankings y en su lugar se utilizó el Webometrics directamente.

El análisis se llevó a cabo vía AFM sobre los siguientes conjuntos de variables, formados por los indicadores de cada ranking: conjunto de indicadores del Webometrics (Web) con algunos indicadores: Conjunto de indicadores del Scimago (Sci) con ocho indicadores, conjunto de indicadores del Qs con seis indicadores, conjunto del País utilizado solo como suplementario. Posteriormente, se llevó a cabo una clasificación jerárquica con los primeros cuatro factores del AFM. Para todos los cáclculos se utilizó código R, el paquete FactoMineR, y los procedimientos $M F A$ y $H C P C$.

\section{Resultados y discusión}

Los resultados se muestran en dos partes: en la primera se presenta un resumen de los resultados de los análisis separados, una descripción básica de los factores del análisis global, se analizan las relaciones entre los rankings (es decir, entre los conjuntos de indicadores de los rankings) y las relaciones entre estos y el AFM. La segunda parte está dedicada a la clasificación y descripción de las clases.

\subsection{Resultados del AFM}

\section{Análisis separados}

- Ranking Webometrics (Web): La figura11ilustra cómo primer factor acumula el $72 \%$ de la varianza e incluye sus cuatro indicadores, razón por la que puede ser resumido adecuadamente por este indicador sintético. Sin embargo, resulta interesante que el segundo factor contrapone presencia en la web con el indicador de excelencia, desvelando una posible paradoja que indicaría lo contraproducente que puede ser dicha presencia para alcanzar la excelencia (ver figura 1). 


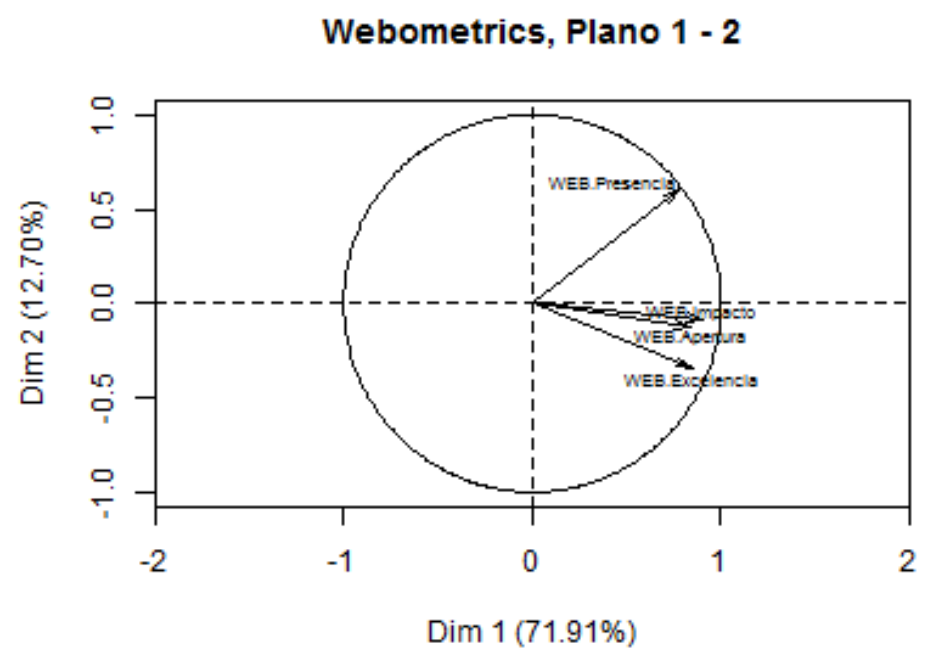

Figura 1: Factores 1 y 2 ranking Web. Fuente: elaboración propia.

- Ranking Scimago (Sci): Se sintetiza en los dos factores mostrados en la figura 2. que incluyen todos sus indicadores y acumulan el $63 \%$ de la varianza. El primero da cuenta del $44 \%$ de la varianza, está explicado principalmente por los indicadores de excelencia, impacto normalizado y publicaciones alta calidad que aportan el $69 \%$ de la varianza del factor. Si además se incluyen como responsables de este factor los indicadores de colaboración internacional y liderazgo científico se consigue el $97 \%$ de su varianza, aunque éstos dos últimos son compartidos con el segundo factor. Además aportan en direcciones opuestas sobre el plano 1-2, resaltando cierta tensión entre la colaboración internacional y el liderazgo científico confirmada por la correlación negativa entre ellos (-0.56). Este hecho indica lo contraproducente que puede resultar la colaboración internacional, si la universidad no se es el principal contribuidor en la producción institucional (ver figura 2). 


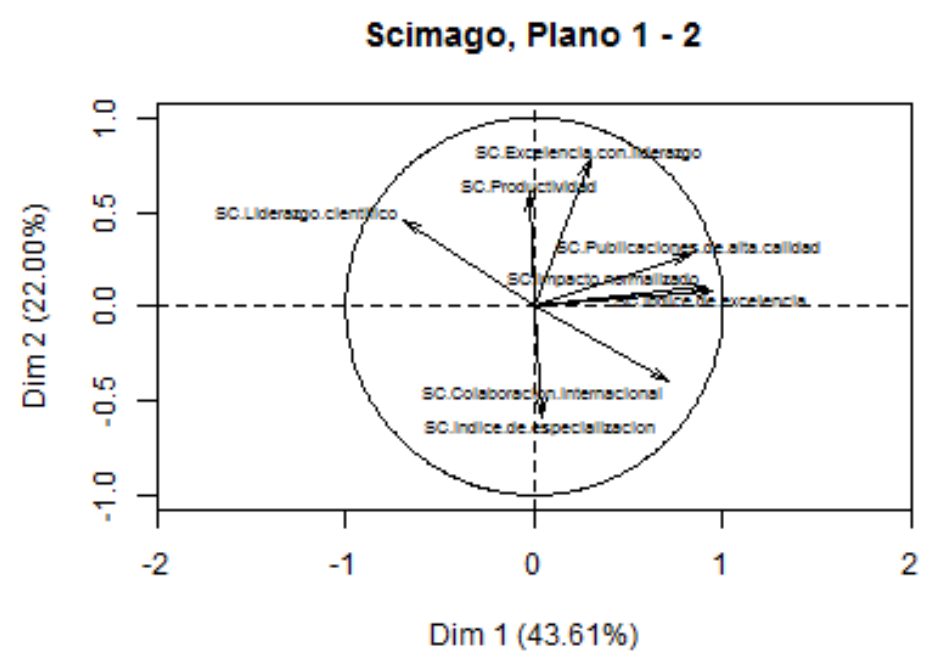

Figura 2: Factores 1 y 2 ranking Sci. Fuente: elaboración propia.

El segundo factor que explica el $22 \%$ de la varianza está tipificado en su parte positiva por excelencia con liderazgo y productividad, y en su parte negativa por el índice de especialización en la producción científica, indicando que éste incide en contra de la excelencia con liderazgo, y en contra de la productividad, aunque esta última relación negativa puede explicarse dado que la producción especializada puede tender a ser menor dependiendo del tamaño del grupo de investigadores de una área determinada.

- Ranking Qs: Un primer análisis indicaría que puede ser sintetizado en dos factores que explican el $58 \%$ de la varianza (ver figura 3 . El primero identifica y asocia la reputación institucional (entre pares académicos y entre empleadores) con el número de docentes con doctorado. El segundo está dominado por el promedio de artículos por docente y, nuevamente, por los dos tipos de reputación institucional que aporta en proporciones similares que el primer factor. Los haces de vectores son casi perpendiculares muestran la independencia entre los correspondientes indicadores (ver figura 3 .

En el anterior análisis, sin embargo, quedan por fuera el número de estudiantes por profesor que tipifica casi en su totalidad al tercer factor, así como el número de citas por artículo, el cual tipifica completamente el cuarto factor, reuniendo entre los cuatro factores el $90 \%$ de la varianza de toda la nube. El anterior análisis es indicador del amplio espectro de criterios tenidos en cuenta en este ranking, al punto en que no es posible reducirlo a dos factores sin perder la valiosa información de la densidad estudiantil y el impacto de la producción institucional medido a través de las citas por artículo (ver figura 4). 


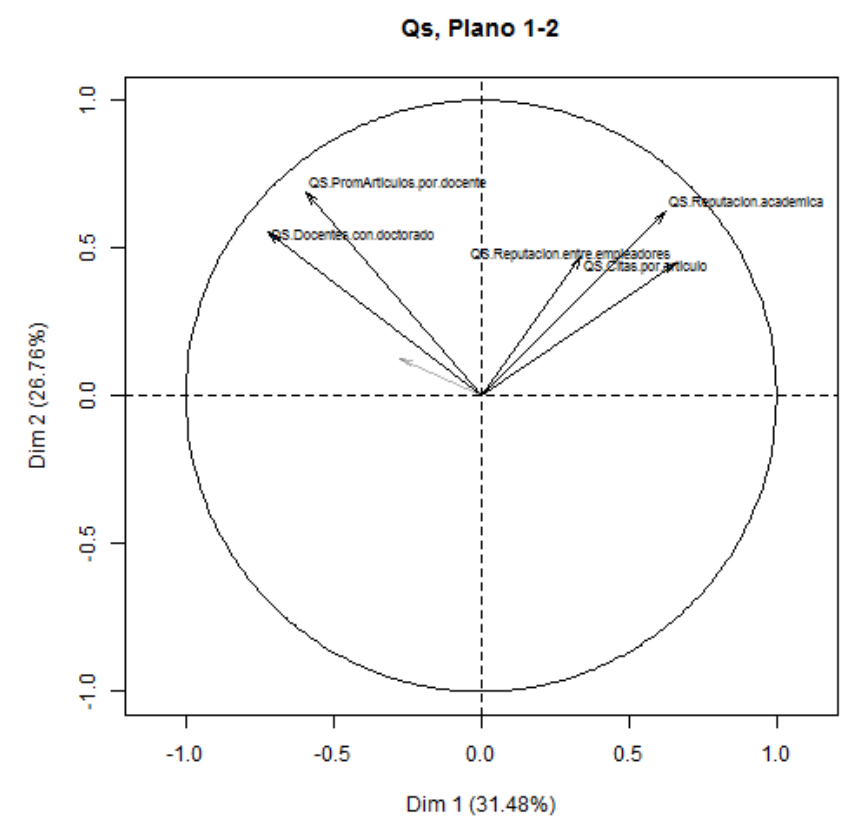

Figura 3: Factores 1 y 2 Ranking Qs. Fuente: elaboración propia.

\section{Análisis global}

Para la interpretación se utilizan las correlaciones variable - factor como principal criterio de inclusión y de nominación del factor, dado que permiten identificar en todos los casos al menos una variable que correlaciona negativamente con este, indicando su direccionalidad y el significado de sus valores negativos. Como en todos los casos la parte positiva del factor fue la que tuvo más variables aportantes, los nombres de los factores se asignan dando prioridad a ésta. Se interpretan los primeros cuatro factores del análisis global que acumulan el $68 \%$ de la varianza y estos mismos se utilizan los cuatro para la clasificación.

- Factor 1: Excelencia, presencia en la web y productividad $\longleftrightarrow$ especialización en la producción científica. En este factor se combinan, en la zona de valores positivos, los cuatro indicadores del Webometrics con el indicador de productividad institucional (Scimago) y productividad docente (Qs). A la zona de valores negativos contribuye, correlaciona y está bien representado el indicador de especialización de la producción científica del Scimago, indicando cierta tensión con la presencia en la web y la productividad. El indicador de especialización es compartido con el factor 4, pero en este no se encuentra bien representado (ver eje horizontal en el figura 5). 


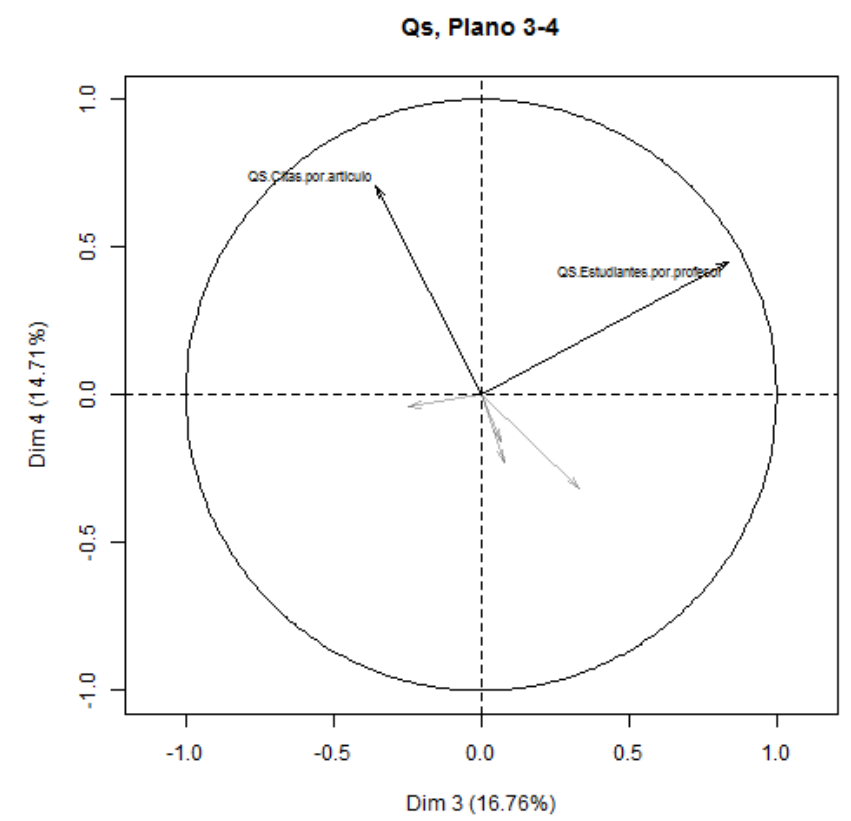

Figura 4: Factores 3 y 4 ranking Qs. Fuente: elaboración propia.

- Factor 2: Colaboración internacional, excelencia e impacto institucional $\longleftrightarrow$ Liderazgo científico. En su zona de valores positivos está tipificado por cuatro indicadores de del Scimago: colaboración internacional, índice de excelencia, impacto normalizado publicaciones de alta calidad y por el indicador de citas por artículo del QS. En la zona de valores negativos se contrapone el indicador de liderazgo científico del ranking Scimago con aporte modesto cercano a la mitad de lo que aportan los del lado positivo, y no muy bien representado. (ver eje vertical en el figura 5)

- Factor 3: Reputación, liderazgo $\longleftrightarrow$ Impacto. Este factor está caracterizado casi en su totalidad en su parte positiva por los dos indicadores de reputación del QS mientras que su parte negativa está débilmente definida por el indicador de impacto normalizado del Scimago. Este hecho indica cierta tensión entre la reputación institucional y el impacto normalizado (ver plano 1-3 en la figura 6). Sin embargo, en el plano 2-3 de la misma figura se nota que aunque sí hay cierta tensión, en realidad reputación e impacto son prácticamente independientes, lo cual se corrobora por las correlaciones entre ellos: $r_{\text {RepPares Aca } \times \text { impacto }}=0.12, r_{\text {RepEmpl } \times \text { impacto }}=0.09$. 


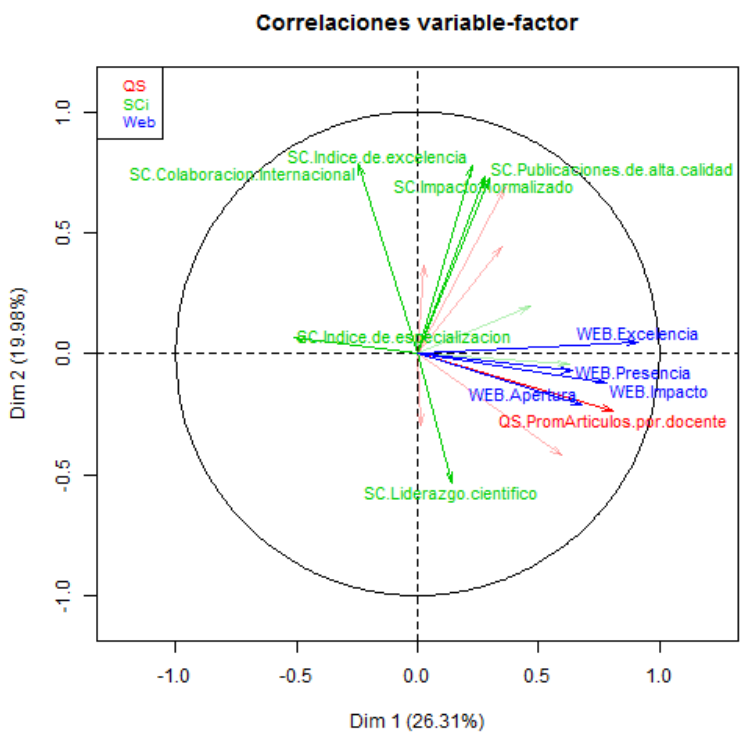

Figura 5: Factores 1 y 2 del análisis global. Fuente: elaboración propia.
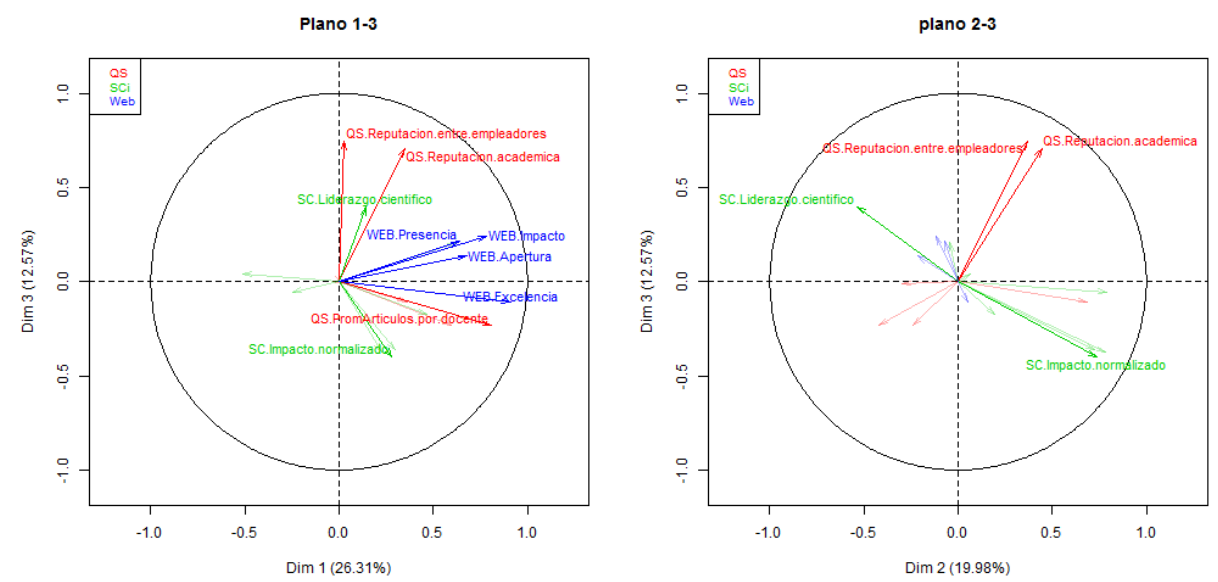

Figura 6: Factor 3 (vertical) del análisis global. Fuente: elaboración propia.

- Factor 4: Capital humano, especialización $\longleftrightarrow$ Baja presencia en web. En este factor se correlaciona en un alto grado con su parte positiva el indicador de estudiantes por profesor (0.8), el índice de especialización (0.44) y el indicador de docentes con doctorado (0.33), y en su parte negativa con todos los indicadores de impacto presencia en la web (ver figura 7).

Comunicaciones en Estadística, junio 2017, Vol. 10, No. 1 


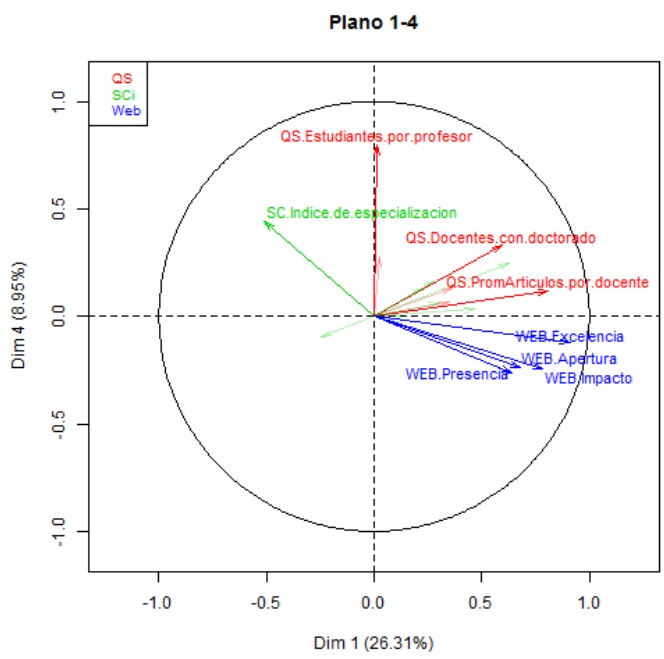

Figura 7: Factor 4 (vertical) del análisis global. Fuente: elaboración propia.

El hecho de que tanto el Webometrics como el Scimago definan o tipifiquen un factor del análisis global, significa que miden conceptos relativamente independientes. Por otra parte, como los indicadores del Qs correlacionan tanto con los indicadores del web como con los del Scimago y también aportan a la tipificación de los cuatro factores se puede interpretar como indicador de la diversidad o dispersión de sus criterios.

Conviene hacer advertir que las correlaciones entre los factores interpretados en los análisis separados de los tres conjuntos de variables: $R_{S c i 2 \times W e b 1}=$ $0.58, R_{Q s 2 \times W e b 1}=0.56 R_{S c i 2 \times Q s 2}=0.53$, y $R_{S c i 1 \times Q s 3}=-0.32$ indican que los criterios utilizados en la construcción de los rankings son medianamente o poco relacionados y que por esta razón resultan las instituciones clasificadas de maneras disímiles en muchos casos. Lo anterior explica por qué son bajas las correlaciones (de Spearman) entre las posiciones ocupados por las universidades en dos de los rankings: $R_{(W e b, Q s)}=0.55, R_{(S c i, Q s)}=0.46$; la correlación alta $R_{(W e b, S c i)}=0.85$ es fácilmente explicable porque los dos rankings utilizan cada uno criterios del otro.

\section{Dimensionalidad y homotecia (coeficientes $L g$ )}

Estas dos características se miden por el coeficiente $L g$ que es indicador del grado de similitud o deformación con respecto a un foco (homotecia) entre los conjuntos de indicadores, y cuando se calcula para un solo conjunto de ellos. Esto se conoce como indicador de la dimensionalidad (Definición en el anexo). El valor del coeficiente $L g_{(\mathrm{Web})}=1.05$ para el Webometrics indica claramente que es de dimensionalidad uno; es decir, que puede sintetizarse en un solo factor; $L g_{(S c i)}=1.4$ indica 
que el Scimago podría ser de dimensionalidad uno o dos, y el valor $L g_{(Q s)}=2.3$ indica que el Qs tiene dos y hasta tres dimensiones o factores que lo caracterizan.

Los coeficientes $L g$ entre conjuntos de indicadores de los rankings muestran por ejemplo que el $Q s$ podría compartir parcialmente un factor con el $S c i L g_{(Q s, S c i)}=$ 0.43 , y con el Webometrics $L g_{(Q s, W e b)}=0.37$, mientras que $S c i$ y web no son conjuntos muy parecidos pues $L g_{(S c i, W e b)}=0.22$.

Adicionalmente, los coeficientes $L g$ de los tres conjuntos con el análisis factorial múltiple (AFM): $L g_{(A F M, Q s)}=1.71$ indica que el $Q s$ comparte hasta dos factores con el factorial múltiple; $L g_{(A F M, S c i)}=1.14$ y $L g_{(A F M, W e b)}=0.92$ indican que $S c i$ y web comparten un factor con el AFM. Finalmente el coeficiente $L g_{(A F M)}=2.09$ indica que éste se puede sintetizar como mínimo en dos factores.

Los coeficientes $l g$ con la variable suplementaria $L g_{(P a i s, Q s)}=0.98, L g_{(P a i s, S c i)}=$ 0.67 y $L g_{(P a i s, W e b)}=0.33$ muestran que ésta podría compartir a lo más un factor con el grupo $Q s$. Por otra parte con el AFM el coeficiente $L g_{A F M, P a i s}=1$ también es uno, indicando que la variable "paísçomparte un factor con el aquel.

\section{Asociación entre los conjuntos de indicadores (Coeficientes Rv)}

La asociación entre conjuntos de indicadores se mide con el coeficiente $R v$ que es una extensión a matrices del coeficiente de correlación de Spearman (definición en el anexo). Los coeficientes $R v_{(Q s, S c i)}=0.24, R v_{(Q s, W e b)}=0.24 \mathrm{y} R v_{(S c i, W e b)}=$ 0.19 indican que entre los indicadores que entre las tres matrices formadas por las universidades con los tres rankings no hay asociaciones considerables, lo cual significa que los criterios que utilizan los tres rankings incluyan temáticas poco asociadas entre ellas mismas.

Por otra parte,los coeficientes entre los conjuntos y el $A F M R v_{(A F M, Q s)}=0.78$, $R v_{(A F M, Q s)}=0.66 \mathrm{y} R v_{(A F M, W e b)}=0.61$ indican que aún no siendo parecidos entre ellos, los tres rankings sí tienen un grado considerable de asociación con el AFM. Es decir, que su representación sobre los planos generados por el AFM es adecuada.

\section{Relaciones de los factores de los análisis separados con los del AFM}

Con el factor 1 del AFM correlacionan mayoritariamente el factor 1 del Webometrics, (impacto presencia institucional en la web), el factor 2 del $Q s$ ( artículos por docente, reputación entre pares académicos y docentes con doctorado) y el factor 2 del Scimago (excelencia-liderazgo-productividad). Los valores extremos positivos de este factor se pueden asociar con presencia-impacto en la web, excelencia, liderazgo, productividad y reputación (ver figura 8). 
Con el factor 2 del AFM correlaciona en primer lugar el factor 1 del Simago (excelencia - impacto - publicaciones de alta calidad y colaboración internacional) seguido por el factor 1 del Qs (reputación entre empleadores y pares académicos). De esta forma, los valores positivos de este factor son indicadores de características deseables para una universidad, acompañados generalmente de buena reputación.

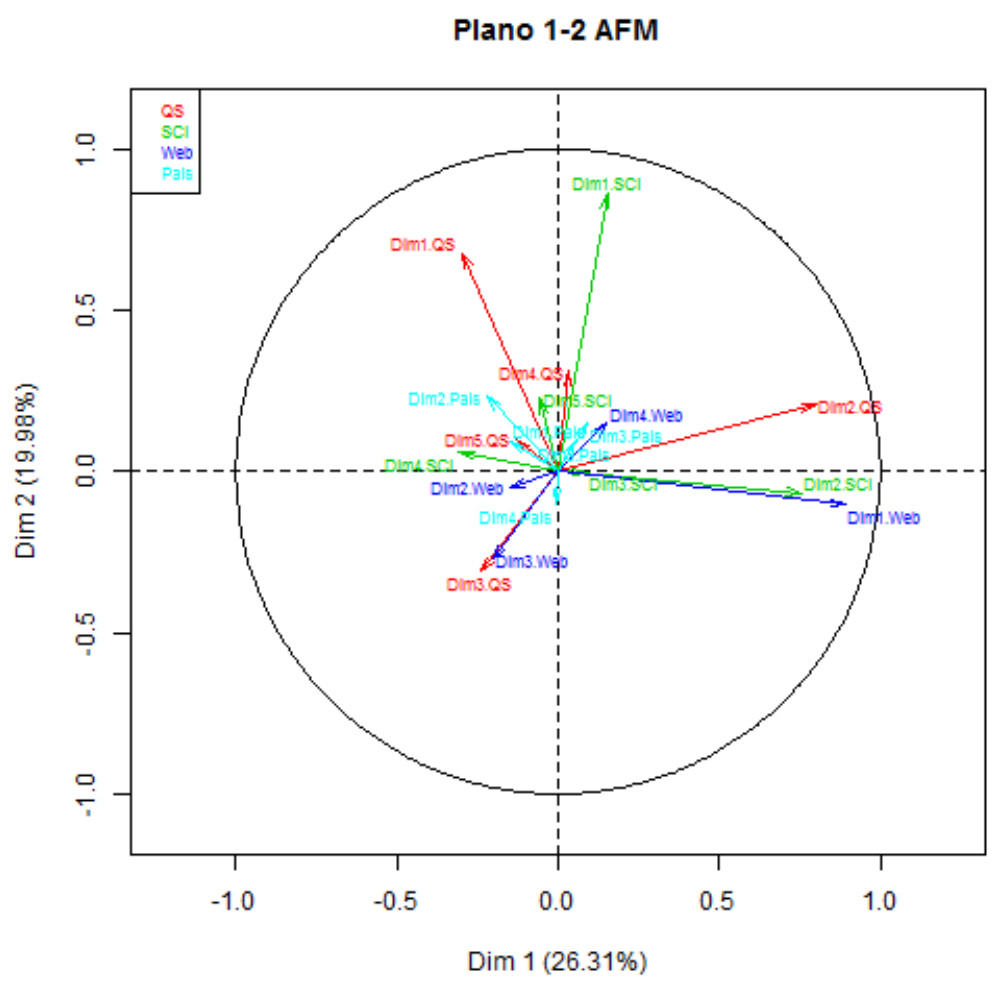

Figura 8: Proyección de los ejes de los análisis parciales sobre los factores 1 y 2 del AFM. Fuente: elaboración propia.

Con el Factor 3 del AFM la mayor correlación es del factor 1 del Qs, razón por la que los valores positivos de este factor son indicadores de reputación institucional y formación docente. Para el factor 4 del AFM, el principal contribuidir es el factor 3 del Qs, que es indicador de la densidad estudiantil de la universidad. De lo anterior se deduce que la influencia del ranking Qs es suficiente para dominar dos de los factores del AFM y corrobora la amplitud del espectro abarcado por sus indicadores en su particular construcción del ranking de universidades.

Comunicaciones en Estadística, junio 2017, Vol. 10, No. 1 


\section{Relaciones de los conjuntos de variables (rankings) con el AFM}

Con cualquiera de los criterios que se utilice (coordinación, contribución, cos2, correlación), el Webometrics asocia casi exclusivamente con el primer factor. El Scimago, por su parte, tiene los mayores valores de todos los criterioscon el factor 2 y se puede asociar moderadamente con el factor 1 por su, también, alta correlación con éste. El Qs, por su parte, tiene cosenos cuadrados bastante bajos en todos los factores (no se encuentra bien representado en ninguno de ellos) y sus otros tres indicadores son ligeramente más altos con el factor 3. En síntesis, el grupo $w e b$ es dominante de manera exclusiva en el factor 1: Mientras que el grupo Sci domina sobre el factor 2 y está compartido con el factor 1. Mientras que el $Q s$ no se puede asociar claramente con ninguno de los factores del AFM como dominante.

\subsection{Resultados de la clasificación}

Para la tipificación de las clases construidas se calculan para cada indicador de cada ranking los siguientes dos indicadores de comparación del promedio de clase $\left(\bar{x}_{\text {clase }}\right)$ con respecto al promedio del grupo completo $\bar{X}$, y de las desviación estándar $\left(\sigma_{\text {clase }}\right)$ de la clase con la desviación estándar del grupo completo $\sigma$ de las 150 universidades del ejercicio:

\section{Indicador de promedio de clase respecto al promedio general:}

$$
I_{\bar{x}}=\frac{\bar{x}_{\text {clase }}}{\bar{X}}
$$

que indica si una clase supera $\left(I_{\bar{x}}>1\right)$ o no $\left(I_{\bar{x}}<1\right)$ el promedio general de todas las universidades analizadas.

Indicador de desviación estándar de la clase respecto a la desviación estándar general

$$
I_{S d}=\frac{\sigma_{\text {clase }}}{\sigma}
$$

que indica si una clase es más dispersa $I_{S d}>1$ o más homogénea $I_{S d}<1$ que el grupo completo de universidades analizadas.

En cada clase se muestran los valores de $I_{\bar{x}}$ e $I_{S d}$ para aquellos indicadores de rankings para los cuales el valor test

$$
\frac{\bar{x}_{\text {clase }}-\bar{X}}{\sigma_{\text {clase }}}>2
$$

Clase 1. Universidades muy especializadas, con buena reputación entre empleadores, alta densidad estudiantil y baja productividad: Las universidades de esta 
clase tienen promedios por encima del promedio del grupo completo de las 150 universidades, en los indicadores de especialización, reputación entre empleadores y número de estudiantes por profesor, y tienen promedios por debajo del grupo de las 150 en productividad (de Scimago), artículos por docente (Qs) y publicaciones en revistas de alto impacto (de Webometrics). En cuanto a su dispersión, esta clase es en general menos dispersa que el grupo completo principalmente en productividad institucional (SCi) y por docente (Qs).

Tabla 1: Indicadores de promedio de la clase 1. Fuente: elaboración propia.

\begin{tabular}{lrr}
\hline & $I_{\bar{x}}$ & $I_{S d}$ \\
\hline SC.Indice.de.especializacion & 1.71 & 1.01 \\
QS.Reputacion.entre.empleadores & 1.22 & 0.74 \\
QS.Estudiantes.por.profesor & 1.19 & 1.06 \\
SC.Publicaciones.de.alta.calidad & 0.80 & 1.05 \\
SC.Impacto.normalizado & 0.79 & 0.79 \\
WEB.Apertura & 0.76 & 1.04 \\
SC.Indice.de.excelencia & 0.74 & 0.81 \\
WEB.Impacto & 0.74 & 0.91 \\
WEB.Presencia & 0.72 & 1.05 \\
QS.Citas.por.articulo & 0.72 & 0.89 \\
SC.Excelencia.con.liderazgo & 0.68 & 0.98 \\
QS.Docentes.con.doctorado & 0.65 & 0.82 \\
WEB.Excelencia & 0.49 & 0.75 \\
QS.PromArticulos.por.docente & 0.27 & 0.32 \\
SC.Productividad & 0.12 & 0.10 \\
\hline
\end{tabular}

De las 38 universidades de esta clase el $29 \%$ son argentinas como se ve en la tabla 2 ;

Tabla 2: Número de universidades por país de la clase 1. Fuente: elaboración propia.

\begin{tabular}{|c|c|c|c|c|c|c|c|c|c|c|}
\hline & 1 & 2 & 3 & 4 & 5 & 6 & 7 & 8 & 9 & 10 \\
\hline Pais & ARG & $\mathrm{COL}$ & $\mathrm{CHL}$ & MEX & VEN & BRA & PAN & PER & URY & CRI \\
\hline Freq & 11 & 6 & 5 & 4 & 3 & 2 & 2 & 2 & 2 & 1 \\
\hline
\end{tabular}

Con el objeto de resaltar características extremas de las clases se calculan para los indicadores de ranking que se obtienen, por ejemplo, para el indicador de especialización "Scimago SC.Indice.de.especializacion" y se mantiene con el mismo nombre pra facilitar la notación en una universidad cualquiera de la clase así:

$$
\text { SC.Indice.de.especializacion }=\frac{\text { SC.Indice.de.especializacion }}{\bar{x}_{\text {clase en Indice.de.especialización }}}
$$


Análogamente, por ejemplo, el idicador de promedio para "SC.Productividad" se calcula así:

$$
\text { SC.Productividad }=\frac{\text { SC.Productividad }}{\bar{x}_{\text {SC.Productividad }}}
$$

Estos indicadores se calculan en adelante para todas las clases y para las cinco universidades que tienen los mayores valores del indicador $I_{\bar{x}}$ de la clase.

Las universidades con mayores índices de especialización comparados con el promedio de la clase 1 y sus correspondientes indicadores de promedio de productividad se muestran en la tabla 3 .

Tabla 3: Las más especializadas del grupo 1. Fuente: elaboración propia.

\begin{tabular}{lrr}
\hline & SC.Indice.de.especializacion & SC.Productividad \\
\hline UNIVERSIDADDECIENCIASEMPRESARIALESYSOCIALES(UCES) & 1.65 & 0.03 \\
- ARG & 1.63 & 0.22 \\
EXTERNADO DE COLOMBIA - COL & 1.58 & 0.42 \\
TORCUATO DI TELLA - ARG & 1.58 & 0.54 \\
COLEGIO DE MEXICO - MEX & 1.58 & 0.47 \\
ALBERTO HURTADO - CHL & & \\
\hline
\end{tabular}

Universidades colombianas en la clase 1: las seis universidades colombianas que se encuentran en esta clase son privadas y se muestran en la tabla 4 junto con cinco de los indicadores $I_{\bar{x}}$ por los que se distingue esta clase:

Tabla 4: Universidades colombianas en la clase 1. Fuente: elaboración propia.

\begin{tabular}{lllllll}
\hline & Sc.Espcial & \multicolumn{2}{l}{ Sc.Productv } & Qs.Reput.Empl & Qs.EstxProf Qs.ArtxDoce \\
\hline PONTIFICIA BOLIVARIA- & 0.07 & 1.63 & 1.04 & 0.64 & 1.43 \\
NA - COL & & & & & \\
EAFIT - COL & 0.29 & 1.03 & 1.07 & 0.45 & 2.02 \\
LA SABANA - COL & 0.79 & 0.80 & 1.28 & 0.76 & 1.37 \\
LA SALLE - COL & 1.15 & 0.36 & 0.99 & 0.37 & 0.29 \\
JORGETADEOLOZANO - & 1.33 & 0.41 & 1.09 & 0.54 & 0.77 \\
COL & & & & & \\
EXTERNADO DE CO- & 1.63 & 0.22 & 1.25 & 0.82 & 0.32 \\
LOMBIA - COL & & & & & & \\
\hline
\end{tabular}

En las dos primeras columnas se nota cómo disminuye el indicador de productividad Sc.Productv con el aumento en el indicador de especialización. Además se observa que la EAFIT, UPB y La Sabana superan el promedio de las 150 analizadas en el indicador de número de artículos por docente (Qs.ArtxDoce) y al mismo tiempo son las de menores índices de especialización, mientras que, por otra parte, las tres con mayores índices de especialización que son el Externado, la Jorge Tadeo Lozano y La Salle son las que tienen los menores índices de artículos por docente (Qs.ArtxDoce) y productividad institucional (Sc.Productv). Lo anterior corrobora, al menos para las universidades colombianas, el tipo de tensión que ya se había notado en el primer factor del análisis global. 
Clase 2. Universidades de excelencia, impacto, colaboración internacional, citaciones por artículo, publicaciones de alta calidad y bajo liderazgo científico Las universidades de esta clase duplican en el índice de excelencia al promedio de las 150, y sus indicadores de impacto, colaboración internacional, citas por artículo, publicaciones de alta calidad e índice de especialización están entre el $41 \%$ y el $76 \%$ por encima de los del grupo de las 150 analizadas. Entre sus debilidades destacan el indicador de liderazgo científico y tres de los cuatro indicadores del Webometrics. La clase es más dispersa que el grupo de las 150 en los indicadores de excelencia, impacto normalizado y liderazgo científico, indicando que algunas de las universidades de esta clase pueden tener altos estos tres indicadores.

Tabla 5: Indicadores de promedio de la clase 2. Fuente: elaboración propia.

\begin{tabular}{lrr}
\hline & $I_{\bar{x}}$ & $I_{S d}$ \\
\hline SC.Indice.de.excelencia & 2.03 & 1.48 \\
SC.Impacto.normalizado & 1.76 & 1.48 \\
SC.Colaboracion.Internacional & 1.73 & 0.85 \\
QS.Citas.por.articulo & 1.58 & 0.78 \\
SC.Publicaciones.de.alta.calidad & 1.49 & 0.87 \\
SC.Indice.de.especializacion & 1.41 & 1.00 \\
WEB.Impacto & 0.87 & 0.69 \\
WEB.Apertura & 0.84 & 1.10 \\
WEB.Presencia & 0.84 & 0.95 \\
SC.Liderazgo.cientifico & 0.69 & 1.46 \\
\hline
\end{tabular}

En esta clase hay no hay ningún país dominante como se ve en la siguiente tabla:

Tabla 6: Número de universidades por país de la clase 2. Fuente: elaboración propia.

\begin{tabular}{lllllllllll}
\hline & 1 & 2 & 3 & 4 & 5 & 6 & 7 & 8 & 9 & 10 \\
\hline Pais & CHL & ECU & MEX & URY & ARG & BOL & COL & PER & PRI & PY \\
Freq & 3 & 2 & 2 & 2 & 1 & 1 & 1 & 1 & 1 & 1 \\
\hline
\end{tabular}

Las cinco universidades con mayor índice de excelencia en esta clase junto con los valores de los indicadores de promedio dentro de la clase son:

Tabla 7: Las excelentes de la clase 2. Fuente: elaboración propia.

\begin{tabular}{lll}
\hline & SC.excelencia & SC.Lid.cientifico \\
\hline IBEROAMERICANA (UIA) - MEX & 1.86 & 0.76 \\
SAN FRANCISCO DE QUITO - ECU & 1.84 & 0.34 \\
DEL NORTE - COL & 1.35 & 0.00 \\
TECNICA FEDERICO SANTA MARIA - CHL & 1.23 & 1.26 \\
MONTEVIDEO - URY & 1.12 & 1.10 \\
\hline
\end{tabular}

Universidades colombianas en la clase 2: en este grupo solo se encuentra la Universidad del Norte con puntajes por encima los del grupo completo en excelencia, impacto y colaboración internacional, y con alto índice de especialización. 
Tabla 8: Universidades colombianas en la clase 2 Indicadores $I_{\bar{x}}$. Fuente: elaboración propia.

\begin{tabular}{rrrrrrr}
\hline & Ind Excel & Impac Nor & Colab Int & Citas x Art & Publ calid & Specializa \\
\hline DEL NORTE - COL & 1.35 & 1.21 & 1.31 & 0.26 & 0.65 & 2.16 \\
\hline
\end{tabular}

Clase 3. Universidades con docentes productivos y formación doctoral, producción de alto impacto y valores bajos en los indicadores de reputación. Se puede tipificar porque el promedio de artículos por docente y el número de docentes con doctorado se encuentran un 38 y $35 \%$ por encima del grupo completo de universidades, pero no gozan de buena reputación entre académicos ni empleadores. Esta clase es más homogénea en todo que el grupo completo, indicando que las universidades pertenecientes a ella son muy parecidas entre sí, en todos los criterios utilizados por los tres rankings.

Tabla 9: Indicadores de promedio de la clase 3. Fuente: elaboración propia.

\begin{tabular}{lrr}
\hline & $I_{\bar{x}}$ & $I_{S d}$ \\
\hline QS.PromArticulos.por.docente & 1.38 & 0.75 \\
QS.Docentes.con.doctorado & 1.35 & 0.71 \\
WEB.Excelencia & 1.10 & 0.41 \\
WEB.Apertura & 1.09 & 0.54 \\
SC.Publicaciones.de.alta.calidad & 0.90 & 0.60 \\
SC.Impacto.normalizado & 0.88 & 0.46 \\
SC.Indice.de.excelencia & 0.81 & 0.43 \\
QS.Citas.por.articulo & 0.75 & 0.63 \\
SC.Indice.de.especializacion & 0.69 & 0.61 \\
SC.Colaboracion.Internacional & 0.67 & 0.65 \\
QS.Reputacion.entre.empleadores & 0.59 & 0.78 \\
QS.Reputacion.academica & 0.54 & 0.59 \\
\hline
\end{tabular}

En esta clase, dominada por universidades brasileras, solo hay universidades de cinco países:

Tabla 10: Número de universidades por país de la clase 3. Fuente: elaboración propia.

\begin{tabular}{llllll}
\hline & 1 & 2 & 3 & 4 & 5 \\
\hline Pais & BRA & MEX & CHL & ARG & VEN \\
Freq & 27 & 7 & 5 & 2 & 1 \\
\hline
\end{tabular}

Las cinco mejores universidades de esta clase muestran un considerable aumento en la reputación con un moderado descenso en el promedio de artículos por docente, como se observa en la tabla 11 
Tabla 11: Las de docentes más productivos de la clase 3. Fuente: elaboración propia.

\begin{tabular}{lrr}
\hline & QS.PromArt.x.docente & QS.Rep.academica \\
\hline FEDERAL DE LAVRAS - BRA & 1.64 & 0.59 \\
ESTADUAL DO NORTE FLUMINENSE - BRA & 1.63 & 0.22 \\
FEDERAL DE VIÇOSA - BRA & 1.63 & 1.10 \\
LA FRONTERA (UFRO) - CHL & 1.59 & 1.46 \\
FEDERAL DO PERNAMBUCO - BRA & 1.54 & 2.14 \\
\hline
\end{tabular}

Universidades colombianas en la clase 3: en este grupo no hay ninguna universidad colombiana.

Clase 4. Universidades con buena reputación entre académicos y empleadores, con impacto por el número de citas, presencia en la web, colaboración internacional, poco especializadas, bajo índice de docentes con doctorado y poca densidad estudiantil. Este grupo es bastante más homogéneo que el grupo completo de universidades analizadas, y en el destaca moderadamente el indicador de reputación entre pares académicos.

Tabla 12: Indicadores de promedio de la clase 4. Fuente: elaboración propia.

\begin{tabular}{lrr}
\hline & $I_{\bar{x}}$ & $I_{S d}$ \\
\hline QS.Reputacion.academica & 1.34 & 0.61 \\
QS.Citas.por.articulo & 1.19 & 0.81 \\
WEB.Presencia & 1.18 & 0.39 \\
QS.Reputacion.entre.empleadores & 1.17 & 0.73 \\
SC.Colaboracion.Internacional & 1.14 & 0.66 \\
WEB.Excelencia & 1.14 & 0.45 \\
WEB.Impacto & 1.13 & 0.53 \\
WEB.Apertura & 1.12 & 0.47 \\
QS.Estudiantes.por.profesor & 0.70 & 0.72 \\
QS.Docentes.con.doctorado & 0.66 & 0.75 \\
SC.Indice.de.especializacion & 0.59 & 0.58 \\
\hline
\end{tabular}

En esta clase, como en la clase uno, también hay mayoría de universidades argentinas:

Tabla 13: Número de universidades por país de la clase 4. Fuente: elaboración propia.

\begin{tabular}{llllllllll}
\hline & 1 & 2 & 3 & 4 & 5 & 6 & 7 & 8 & 9 \\
\hline Pais & ARG & MEX & COL & CHL & VEN & CRI & PER & CUB & ECU \\
Freq & 10 & 6 & 5 & 4 & 3 & 2 & 2 & 1 & 1 \\
\hline
\end{tabular}


Las cinco mejores universidades de este grupo son:

Tabla 14: Las de mejor reputación académica de la clase 4. Fuente: elaboración propia.

\begin{tabular}{lll}
\hline & QS.Reputacion.academica & SC.Indice.de.especializacion \\
\hline BUENOS AIRES - ARG & 1.26 & 0.94 \\
NACIONAL DE LA PLATA - ARG & 1.25 & 0.10 \\
PONTIFICIA JAVERIANA - COL & 1.24 & 0.37 \\
NACIONAL DE CORDOBA - ARG & 1.24 & 0.63 \\
SANTIAGO DE CHILE (USACH) - & 1.24 & 1.62 \\
CHL & & \\
\hline
\end{tabular}

Universidades colombianas en el grupo 4: en este grupo se encuentran tres universidades públicas y dos privadas:

Tabla 15: Universidades colombianas de la clase 4. Fuente: elaboración propia.

\begin{tabular}{llllll}
\hline & $\begin{array}{l}\text { ANTIOQUIA } \\
\text { COL }\end{array}$ & $\begin{array}{l}\text { PONTIFICIA } \\
\text { JAVERIA- } \\
\text { NA - COL }\end{array}$ & $\begin{array}{l}\text { DEL ROSA- } \\
\text { RIO - COL }\end{array}$ & $\begin{array}{l}\text { DEL VALLE } \\
\text { - COL }\end{array}$ & $\begin{array}{l}\text { INDUSTRIAL } \\
\text { DE } \\
\text { TANDER } \\
\text { SAN- } \\
\text { COL }\end{array}$ \\
\hline Rep Aca & 1.19 & 1.24 & 0.82 & 1.10 & 0.77 \\
Qs Cit x Art & 1.19 & 0.60 & 1.31 & 0.59 & 0.89 \\
Web Presen & 1.01 & 0.99 & 1.08 & 1.00 & 0.84 \\
Qs REp Empl & 1.19 & 1.40 & 1.34 & 0.86 & 1.03 \\
Sc Col Int & 1.11 & 0.98 & 0.86 & 1.08 & 1.01 \\
Web Excel & 1.13 & 0.92 & 0.88 & 0.99 & 0.96 \\
Web impct & 1.05 & 1.02 & 0.69 & 0.92 & 0.75 \\
Web Apert & 1.06 & 1.01 & 0.90 & 1.03 & 1.04 \\
\hline
\end{tabular}

Se observa que la Universidad de Antioquia tiene la mayoría de sus indicadores por encima del promedio, exeptuando los de reputación entre pares académicos y entre empleadores que son mayores para la Universidad Javeriana.

Clase 5. Universidades de élite: productivas, docentes productivos y con formación doctoral y reputación entre académicos, excelencia y liderazgo y con bajos índices de colaboración internacional y de especialización. En este grupo sobresalen el indicador de productividad del Scimago (SC.Productividad) casi cuadruplica el promedio del grupo de las 150 y el de productividad docente del Qs (QS.PromArticulos.por.docente) duplica el del promedio del grupo completo de universidades analizadas. Además, con excepción de los indicadores de especialización y de colaboración internacional, todos los demás indicadores superan el promedio de todas las universidades. 
Tabla 16: Indicadores de promedio de la clase 5. Fuente: elaboración propia.

\begin{tabular}{lrr}
\hline & $I_{\bar{x}}$ & $I_{S d}$ \\
\hline SC.Productividad & 3.73 & 1.97 \\
QS.PromArticulos.por.docente & 2.00 & 0.53 \\
QS.Docentes.con.doctorado & 1.63 & 0.38 \\
QS.Reputacion.academica & 1.49 & 0.54 \\
WEB.Excelencia & 1.43 & 0.30 \\
SC.Excelencia.con.liderazgo & 1.28 & 0.58 \\
QS.Citas.por.articulo & 1.28 & 0.75 \\
WEB.Impacto & 1.27 & 0.29 \\
QS.Reputacion.entre.empleadores & 1.26 & 0.86 \\
WEB.Presencia & 1.25 & 0.39 \\
QS.Estudiantes.por.profesor & 1.25 & 0.74 \\
WEB.Apertura & 1.20 & 0.28 \\
SC.Colaboracion.Internacional & 0.81 & 0.62 \\
SC.Indice.de.especializacion & 0.70 & 0.66 \\
\hline
\end{tabular}

En esta clase, como en la clase tres, también dominan las universidades brasileñas:

Tabla 17: Número de universidades por país de la clase 5. Fuente: elaboración propia.

\begin{tabular}{lllll}
\hline & 1 & 2 & 3 & 4 \\
\hline Pais & BRA & CHL & COL & MEX \\
Freq & 14 & 3 & 2 & 2 \\
\hline
\end{tabular}

Las mejores universidades del grupo 5 son:

Tabla 18: Las más productivas de la clase 5. Fuente: elaboración propia.

\begin{tabular}{lrr}
\hline & SC.Productividad & SC.Indice.de.especializacion \\
\hline DE SAO PAULO (USP) - BRA & 4.74 & 1.67 \\
NACIONAL AUTONOMA DE ME- & 1.92 & 1.32 \\
XICO (UNAM) - MEX & & \\
UNIVERSIDADE ESTADUALPAU- & 1.71 & 0.00 \\
LISTA "JULIODEMESQUITAFIL- & & 2.24 \\
HO BRA & 1.67 & 2.06 \\
ESTADUAL DE CAMPINAS (UNI- & & \\
CAMP) - BRA & 1.49 & \\
FEDERAL DO RIO DE JANEIRO - & & \\
BRA & &
\end{tabular}

Universidades colombianas en el grupo 5: dos universidades colombianas se encuentran en el grupo élite: 
Tabla 19: Universidades colombianas en el grupo 5 Indicadores $I_{\bar{x}}$. Fuente: elaboración propia.

\begin{tabular}{lllr}
\hline & $\begin{array}{l}\text { LOS ANDES CO- } \\
\text { LOMBIA - COL }\end{array}$ & $\begin{array}{l}\text { NACIONAL } \\
\text { COLOMBIA } \\
\text { COL }\end{array}$ & $\begin{array}{r}\text { DE } \\
\text { CO }\end{array}$ \\
\hline Sc prod & 0.23 & 0.57 \\
Qs Art x Doc & 0.98 & 0.83 \\
Qs Doc Drado & 0.91 & 0.75 \\
Qs Rep Aca & 1.13 & 1.13 \\
Web Excel & 0.96 & 0.94 \\
Sc Excel Lid & 0.73 & 0.71 \\
Qs Cit x Art & 1.33 & 0.39 \\
Web Impct & 0.93 & 1.05 \\
Qs Rep Empl & 1.31 & 1.31 \\
Web Presen & 0.91 & 1.08 \\
Qs Est x Prof & 0.80 & 0.65 \\
Web Aprtur & 0.96 & 1.04 \\
\hline
\end{tabular}

\section{Conclusiones}

Los matices que distinguen la clasificación permiten ver la diversidad de perfiles institucionales y descubrir algunas paradojas sobre los indicadores o criterios incluidos en los rankings utilizados para este ejercicio. Para empezar, el hecho de que en la clase 1 se encuentren universidades con alto índice de especialización y baja productividad, y en la clase 5 las universidades que tienen bajos índices de especialización sean las que tienen altos niveles de productividad, muestra lo contraproducente que puede llegar a ser el grado de especialización para puntuar bien en los indicadores de productividad. Varias interpretaciones son posibles de lo anterior: por ejemplo que el radio de impacto de la producción científica especializada es mucho más reducido que el de universidades con producción menos concentrada en algunos campos temáticos, o que las instituciones que publican en temáticas especializadas son poco productivas.

En la clase 3 se encuentran universidades productivas con docentes que tienen formación de doctorado y con producción de alto impacto, pero de baja reputación. Por esta razón surge la pregunta sobre la pertinencia de indicadores de opinión, como la reputación entre académicos y empleadores del Qs, cuando lo que se pretende es clasificar universidades por su quehacer académico-científico.

Dos paradojas más surgen de las clases 2 y 4: de la clase 2 se concluye que el liderazgo científico, medido por el porcentaje de producción de una institución en la cual este es el principal contribuidor, no necesariamente es un criterio de excelencia o impacto y, adicionalmente, que aun teniendo altos valores en el indicador de colaboración internacional, una institución puede tener publicaciones de alta calidad sin ejercer liderazgo científico. De la clase 4, por otra parte, se deduce que la formación doctoral o la baja densidad estudiantil no necesariamente son factores de reputación, impacto, o colaboración institucional 
El primer factor del análisis global muestra cierta tensión entre el grado de especialización institucional y los indicadores de excelencia, presencia en la web y productividad docente, explicable en parte por el hecho de que altos grados de especialización en un campo temático reduce el círculo de impacto a los investigadores del mismo campo.

El segundo factor evidencia cierta paradoja al contraponer el liderazgo científico (porcentaje de la producción institucional en el que la institución es el principal contribuidor) con los indicadores de impacto, calidad de la producción, producción institucional y por docente, como si el liderazgo fuera contraproducente para puntuar bien en dichos indicadores.

La proyección sobre los factores dos y tres de los indicadores de liderazgo, impacto y reputación institucional muestra una asociación negativa entre los dos primeros, y al mismo tiempo los muestra como dos prácticamente independientes de la reputación institucional.

En el cuarto factor sobresale el hecho de que la densidad estudiantil es un criterio que no altera prácticamente ninguno de los indicadores, con lo que se podría decir que para los rankings universitarios sobre el quehacer académico investigativo no sería de interés al menos para las universidades analizadas.

La dimensionalidad de los tres rankings (uno para el Webometrics, dos para el Simago y cuatro para el Qs) es indicador de la diversidad de los criterios utilizados en los rankings, mientras que las bajas correlaciones entre los factores producidos por los análisis separados son indicadores de que ninguno de ellos clasifica de manera integral a las universidades.

Recibido: 11 de abril de 2016 Aceptado: 16 de agosto de 2016

\section{Referencias}

Escofier, B. P. J. (2008), Analyses Factorielles Simples et Multiples, 4 edn, DUNOD, Paris, ISBN 978-2-10-053809-6.

Escoufier, Y. (1973), 'Le traitement des variables vectorielles', Biometrics 29, 751760.

Herve Abdi, L. J. W. \& Valentin, D. (2013), 'Multiple factor analysis: principal component analysis for multitable and multiblock data sets', WIREs Comput Stat 2013 doi: 10.1002/wics.1246.

Margison, S. \& Ordorika, I. (2010), Hegemonía en la era del conocimiento: competencia global en la educación superior y la investigación científica, 2 edn, Academic Press, Universidad Nacional Autónoma de México.

Martinez Rizo, F. (2011), 'Los rankings de universidades: una visión crÃtica', Revista de la educación superior $\mathbf{X L}(\mathbf{I})(157), 77-97$. 
Sanchez, J. \& Moreno, G. (2011), Caracterización de rankings universitarios, 1 edn, Universidad Nacional de Colombia, sede Bogotá, Universidad Nacional de Colombia, sede Bogotá. 


\section{Anexo 1. Algunos conceptos importantes}

Coeficiente $R v$ : definido por Escoufier (1973). Es una medida de la similitud entre dos matrices semidefinidas positivas (sdp) y corresponde a una extensión para matrices del coeficiente de correlación de Spearman. Sean $X_{N, J}$ y $Y_{N, K}$ matrices reales. Entonces las matrices $S=X X^{\prime}$ y $T=Y Y^{\prime}$ son sdp. El coeficiente $R_{v}$ entre $S$ y $T$ se define por:

$$
R_{v}=\frac{\operatorname{Traza}\left(S^{\prime} T\right)}{\sqrt{\operatorname{Traza}\left(S^{\prime} S\right) \operatorname{Traza}\left(T^{\prime} T\right)}} .
$$

En AFM las matrices tiene el mismo número de filas y por tanto se puede calcular la homotecia también a partir de las matrices de covarianzas $S=X^{\prime} X$ y $T=Y^{\prime} Y$, y por esta razón se puede interpretar como la suma de los productos de las varainzas y covarianzas de cada grupos de variables.

Coeficiente $L g$ : una medida de la relación entre pares de tablas que indica cuántas características son compartidas entre ellas en sentido de homotecia 4 Entre más variables de una de ellas relacionen con las de la otra, más grande es el coeficiente $L g$. Se define por:

$$
L_{g}=\frac{\operatorname{Traza}\left(S^{\prime} T\right)}{\alpha_{1}^{2} \times \lambda_{1}^{2}} .
$$

donde $\alpha_{1}$ y $\lambda_{1}$ es el primer valor propio de $S$ y de $T$ respectivamente. Ver Herve Abdi \& Valentin (2013).

\footnotetext{
${ }^{4}$ Proyección con respecto a un punto de una figura (de homotecia) en proporciones mayores o menores, de forma directa o inversa
} 